\title{
Cosmological model of the interaction between dark matter and dark energy
}

\author{
J. S. Wang ${ }^{1,2}$ and F. Y. Wang ${ }^{1,2}$ \\ 1 School of Astronomy and Space Science, Nanjing University, Nanjing 210093, PR China
2 Key Laboratory of Modern Astronomy and Astrophysics (Nanjing University), Ministry of Education, Nanjing 210093, PR China
e-mail: fayinwang@nju.edu.cn
}

Received 4 September 2013 / Accepted 16 March 2014

\begin{abstract}
We tested a cosmological model for the interaction between dark matter and dark energy with a dynamic equation of state $w_{\mathrm{DE}}(z)=$ $w_{0}+w_{1} z /(1+z)$, using type Ia supernovae (SNe Ia), Hubble parameter data, baryonic acoustic oscillation (BAO) measurements, and cosmic microwave background (CMB) observations. This interacting cosmological model has not been studied before. The best-fit parameters with $1 \sigma$ uncertainties are $\delta=-0.022 \pm 0.006, \Omega_{\mathrm{DM}}^{0}=0.213 \pm 0.008, w_{0}=-1.210 \pm 0.033$ and $w_{1}=0.872 \pm 0.072$ with $\chi_{\min }^{2} /$ d.o.f. $=0.990$. At the $1 \sigma$ confidence level, we find $\delta<0$, which means that the energy transfer prefer ably occurring from dark matter to dark energy. We also find that the SNe Ia data disagree with the combined CMB, BAO, and Hubble parameter data. The evolution of $\rho_{\mathrm{DM}} / \rho_{\mathrm{DE}}$ indicates that this interacting model is a good approach to solve the coincidence problem, because $\rho_{\mathrm{DE}}$ decreases with scale factor $a$. The transition redshift is $z_{\text {tr }}=0.63 \pm 0.07$ in this model.
\end{abstract}

Key words. dark energy - cosmology: observations - cosmological parameters

\section{Introduction}

Recent observations have shown with increasing accuracy that the Universe is undergoing an accelerating expansion. This can bee seen from Type Ia supernovae data ( $\mathrm{SNe}$ Ia; Riess et al. 1998; Perlmutter et al. 1999; Suzuki et al. 2012), cosmic microwave background (CMB) from Wilkinson Microwave Anisotropy Probe 9 years (WMAP9; Hinshaw et al. 2013) and Planck (Planck Collaboration XVI 2014), the baryonic acoustic oscillation (BAO) from 6dF Galaxy Redshift Survey (6dFGRS; Beutler et al. 2011), the Sloan Digital Sky Survey (SDSS; Eisenstein et al. 2005; Percival et al. 2010; Anderson et al. 2012), WiggleZ (Blake et al. 2012) and so on. Planck results also confirm that the Universe is spatially flat, in other words, the curvature parameter $\Omega_{K}$ is $-0.0000_{-0.0067}^{+0.0066}$ (Planck Collaboration XVI 2014) at $95 \%$ confidence level. The main components of the Universe are dark matter (DM) and dark energy (DE). The special characteristic of DE is negative pressure. The simplest candidate for $\mathrm{DE}$ is the cosmological constant with its equation of state $(\operatorname{EoS}) w=p_{\Lambda} / \rho_{\Lambda}=-1$. However, there are some problems with the $\Lambda \mathrm{CDM}$ model. The most important one is the coincidence problem, which describes why the DE density is comparable with the matter density at present. But, the energy density of $\mathrm{DE}$ is non-dynamical, while matter density decreases with $a^{-3}$, where $a=1 /(1+z)$ is scale factor.

To solve the coincidence problem, many methods have been proposed (Ratra \& Peebles 1988; Caldwell 2002; Armendariz-Picon et al. 2001; Feng et al. 2005). The interacting dark sector models are possible solutions, which means that there is an energy exchange between DE and DM, the energy density ratio $\rho_{\mathrm{DM}} / \rho_{\mathrm{DE}}$ can decrease more slowly than $a^{-3}$. We consider that the energy is exchanged through an interaction term $Q$. The conservation of the total stress-energy tensor and a scalar-field model of dark energy is also assumed in this case

$$
\begin{aligned}
& \left(\dot{\rho}_{\mathrm{B}}+\dot{\rho}_{\mathrm{DM}}\right)+3 H\left(\rho_{\mathrm{B}}+\rho_{\mathrm{DM}}\right)=-Q, \\
& \dot{\rho}_{\mathrm{DE}}+3 H \rho_{\mathrm{DE}}\left(1+w_{\mathrm{DE}}\right)=Q,
\end{aligned}
$$

where $\rho_{\mathrm{B}}$ and $\rho_{\mathrm{DM}}$ represent the energy density of baryon and cold dark matter, $\rho_{\mathrm{DE}}$ is the energy density of dark energy with a dynamic $\operatorname{EoS} w_{\mathrm{DE}}$, and $H=\dot{a} / a$ is the Hubble parameter. Many interacting theoretical models have been studied (Amendola 2000; Farrar \& Peebles 2004; Guo et al. 2005; Szydłowski 2006; Sadjadi \& Alimohammadi 2006; Del Campo et al. 2006; Wei \& Cai 2006; Bertolami et al. 2007; Cai \& Su 2010), but the interaction term $Q$ is still poorly known. Many phenomenological models have been put forward to solve it, such as a simple phenomenological coupling form $Q=C \delta(a) H \rho_{\text {DM }}$ (Dalal et al. 2001; Amendola et al. 2007; Guo et al. 2007; Wei 2010b; Cao et al. 2011), where $C$ is constant. The EoS of dark energy is needed to solve Eq. (2). We here discuss a phenomenological model with a dynamic EoS (Chevallier \& Polarski 2001; Linder 2003),

$w_{\mathrm{DE}}(z)=w_{0}+w_{1} z /(1+z)$

Then we calculate the evolution of the energy density of DM and DE. The transition redshift is also constrained in this interacting phenomenological model (Abdel-Rahman \& Riad 2007).

The structure of this paper is arranged as follows: in Sect. 2, we analyze the model. In Sect. 3, we constrain model parameters using the observational data sets. In Sect. 4, we present our results. The conclusions and discussions are given in Sect. 5. 


\section{Interacting dark sector model}

The interaction is between the non-baryonic dark matter and the quintessence field. The mass evolution of dark matter particles can be written as $m=m(\Phi(a))$, and parameterized as a function of the scale factor $\delta(a)$ (Amendola et al. 2007; Majerotto et al. 2004; Rosenfeld 2005),

$m(a)=m_{0} \mathrm{e}_{1}^{a} \delta\left(a^{\prime}\right) \mathrm{d} \ln a^{\prime}$,

where $m_{0}$ is the current dark matter mass and $\delta(a)=\operatorname{dln} m / \operatorname{dln} a$ represents the rate of change of the dark matter mass. We set $\delta(a)$ as a constant in this paper (Amendola et al. 2007).

The evolutions of $\rho_{\mathrm{DM}}$ and $\rho_{\mathrm{DE}}$ can be expressed as

$\dot{\rho}_{\mathrm{DM}}+3 H \rho_{\mathrm{DM}}-\delta H \rho_{\mathrm{DM}}=0$,

$\dot{\rho}_{\mathrm{DE}}+3 H \rho_{\mathrm{DE}}\left(1+w_{\mathrm{DE}}\right)+\delta H \rho_{\mathrm{DM}}=0$.

The interacting term is $Q=-\delta H \rho_{\mathrm{DM}}$. Then Eq. (5) can be solved assuming constant interaction,

$\rho_{\mathrm{DM}}(a)=\rho_{\mathrm{DM}}^{0} a^{-3+\delta}$,

where $\rho_{\mathrm{DM}}^{0}$ is the current dark matter energy density. Substituting this solution into Eq. (6), we obtain the evolution of $\rho_{\mathrm{DE}}$,

$\frac{\mathrm{d} \rho_{\mathrm{DE}}}{\mathrm{d} a}+\frac{3}{a} \rho_{\mathrm{DE}}\left(1+w_{\mathrm{DE}}\right)+\delta \rho_{\mathrm{DM}}^{0} a^{-4+\delta}=0$.

Amendola et al. (2007) studied the interacting model with an EoS $w_{\mathrm{DE}}(z)=w_{0}+w_{1} z$. But this model is not compatible with CMB data since it diverges at high redshift (Chevallier \& Polarski 2001). We considered an extended parameterization of the EoS as Eq. (3) to avoid this problem (Chevallier \& Polarski 2001; Linder 2003). Then we obtain the solution of Eq. (8) as a function of redshift $z$,

$\rho_{\mathrm{DE}}(z)=\rho_{\mathrm{DE}}^{\mathrm{NI}}(z)\left[1+\Theta\left(z, w_{0}, w_{1}, \delta\right)\right]$,

where

$\rho_{\mathrm{DE}}^{\mathrm{NI}}(z)=\rho_{\mathrm{DE}}^{0} \mathrm{e}^{-3 w_{1} z /(1+z)}(1+z)^{3\left(1+w_{0}+w_{1}\right)}$.

This represents the evolution of the dark energy density without interaction for this parameterization. The $\Theta$ function is

$$
\begin{aligned}
\Theta\left(z, w_{0}, w_{1}, \delta\right)= & \delta \mathrm{e}^{3 w_{1}}\left(3 w_{1}\right)^{-3\left(w_{0}+w_{1}\right)-\delta} \times \frac{\rho_{\mathrm{DM}}^{0}}{\rho_{\mathrm{DE}}^{0}} \\
& \times \Gamma\left(3\left(w_{0}+w_{1}\right)+\delta, 3 w_{1} /(1+z), 3 w_{1}\right),
\end{aligned}
$$

where $\Gamma\left(a, x_{0}, x_{1}\right)$ is the generalized incomplete gamma function $\Gamma\left(a, x_{0}, x_{1}\right)=\int_{x_{0}}^{x_{1}} t^{a-1} \mathrm{e}^{-t} \mathrm{~d} t$.

Then the Hubble parameter in this dark interaction model can be written as

$$
\begin{array}{r}
E\left(z, \Omega_{\mathrm{DM}}^{0}, w_{0}, w_{1}, \delta\right)=\left[\Omega_{\mathrm{DM}}^{0}(1+z)^{3-\delta}+\Omega_{\mathrm{B}}^{0}(1+z)^{3}\right. \\
+\left(1-\Omega_{\mathrm{B}}^{0}-\Omega_{\mathrm{DM}}^{0}-\Omega_{\mathrm{r}}^{0}\right)(1+z)^{3\left(1+w_{0}+w_{1}\right)} \\
\left.\times \mathrm{e}^{-3 w_{1} z /(1+z)}\left(1+\Theta\left(z, w_{0}, w_{1}, \delta\right)\right)+\Omega_{\mathrm{r}}^{0}(1+z)^{4}\right]^{1 / 2},
\end{array}
$$

where $\Omega_{\mathrm{DM}}^{0}, \Omega_{\mathrm{B}}^{0}$ and $\Omega_{\mathrm{r}}^{0}$ are the dark matter, the current baryonic, and the radiation density fractions. We adopted $\Omega_{\mathrm{B}}^{0}=$ $0.0487 \pm 0.0006$ (Planck Collaboration XVI 2014), $H_{0}=73.8 \pm$ $2.4 \mathrm{~km} \mathrm{~s}^{-1} \mathrm{Mpc}^{-1}$ (Riess et al. 2011) and $\Omega_{\mathrm{r}}^{0}=\left(\Omega_{\mathrm{DM}}^{0}+\Omega_{\mathrm{B}}^{0}\right) /(1+$ $\left.z_{e q}\right)$, where $z_{e q}$ is the redshift when the matter energy density is equal to the radiation energy density.

\section{Observational data}

To tightly constrain the parameters, we combined an SNe Ia sample, the Hubble parameter data, BAO measurements, and CMB observation. Each one of these data can constrain the cosmological parameter compactly and consistently (Suzuki et al. 2012; Farooq \& Ratra 2013; Hinshaw et al. 2013; Planck Collaboration XVI 2014).

\subsection{SNe la data}

$\mathrm{SNe}$ Ia data samples are the first evidence for the accelerating expansion of the Universe, and they can be taken as standard candles to measure the luminosity distance. We used the latest Union 2.1 sample (Suzuki et al. 2012), which contains $580 \mathrm{SNe}$ Ia in the redshift range $0.014<z<1.415$. With the measured luminosity distance $d_{L}$ in units of megaparsecs, the predicted distance modulus can be given as

$\mu=5 \log \left(d_{L}\right)+25$,

where the luminosity distance is expressed as

$d_{L}\left(z, \Omega_{\mathrm{DM}}^{0}, w_{0}, w_{1}, \delta\right)=c \frac{(1+z)}{H_{0}} \int_{0}^{z} \frac{\mathrm{d} z^{\prime}}{E\left(z^{\prime}, \Omega_{\mathrm{DM}}^{0}, w_{0}, w_{1}, \delta\right)}$.

The likelihood functions can be determined from $\chi_{\mathrm{SNe}}^{2}$ distribution (Nesseris \& Perivolaropoulos 2005; Wang 2012),

$\chi_{\mathrm{SNe}}^{2}=A-\frac{B^{2}}{C}$,

where $A=\sum_{i}^{580}\left(\mu^{\mathrm{obs}}-\mu^{\mathrm{th}}\right)^{2} / \sigma_{\mu, i}^{2}, B=\sum_{i}^{580}\left(\mu^{\mathrm{obs}}-\mu^{\mathrm{th}}\right) / \sigma_{\mu, i}^{2}$, $C=\sum_{i}^{580} 1 / \sigma_{\mu, i}^{2}$. $\mu^{\mathrm{obs}}$ is the observational distance modulus, and $\sigma_{\mu, i}$ is the $1 \sigma$ uncertainty of the distance moduli.

\subsection{Hubble parameter data}

The Hubble parameter sample contains 28 data points, which cover the redshift range $0.07 \leq z \leq 2.3$. This is the largest data set of $H(z)$, with nine data from Simon et al. (2005), two from Stern et al. (2010), eight from Moresco et al. (2012), one from Busca et al. (2013), four from Zhang et al. (2012), three from Blake et al. (2012), and one from Chuang \& Wang (2013). These data have been compiled by (Farooq \& Ratra 2013, see their Table 1). The $\chi_{H}^{2}$ is given as

$\chi_{H}^{2}=\sum_{i=1}^{28} \frac{\left[H\left(z_{i}\right)-H_{\mathrm{obs}}\left(z_{i}\right)\right]^{2}}{\sigma_{h, i}^{2}}$,

where theoretical $H(z)$ can be obtained from Eq. (12), $H_{\text {obs }}$ and $\sigma_{h, i}$ are observed values.

\subsection{Baryon acoustic oscillations}

The BAO peak in the galaxy correlation function was first detected in the 2dFGRS (Cole et al. 2005) and SDSS (Eisenstein et al. 2005). Now the BAO redshift covers the range $0.1 \leq z \leq$ 0.73 . The distance ratio $d_{z}$ is defined as

$d_{z}=\frac{r_{\mathrm{s}}\left(z_{\mathrm{d}}\right)}{D_{V}\left(z_{\mathrm{BAO}}\right)}$,

where the angular diameter distance scale $D_{V}$ is given by Eisenstein et al. (2005),

$D_{V}\left(z_{\mathrm{BAO}}\right)=\frac{1}{H_{0}}\left[\frac{z_{\mathrm{BAO}}}{E\left(z_{\mathrm{BAO}}\right)}\left(\int_{0}^{z_{\mathrm{BAO}}} \frac{\mathrm{d} z}{E(z)}\right)^{2}\right]^{1 / 3}$. 
The comoving sound horizon at the drag epoch is $r_{\mathrm{s}}\left(z_{\mathrm{d}}\right)=$ $H_{0}{ }^{-1} \int_{z_{\mathrm{d}}}^{\infty} c_{\mathrm{s}}(z) / E(z) \mathrm{d} z$. Following Eisenstein \& $\mathrm{Hu}$ (1998), the decoupled redshift is

$$
\begin{aligned}
z_{\mathrm{d}}= & \left\{1291\left(\Omega_{\mathrm{M}}^{0} h^{2}\right)^{0.251} /\left[1+0.659\left(\Omega_{\mathrm{M}}^{0} h^{2}\right)^{0.828}\right]\right\} \\
& \times\left[\left(1+b_{1}\left(\Omega_{\mathrm{B}}^{0} h^{2}\right)^{b_{2}}\right)\right],
\end{aligned}
$$

with

$$
\begin{aligned}
& b_{1}=0.313\left(\Omega_{\mathrm{M}}^{0} h^{2}\right)^{-0.419}\left[1+0.607\left(\Omega_{\mathrm{M}}^{0} h^{2}\right)^{0.674}\right]^{-1}, \\
& b_{2}=0.238\left(\Omega_{\mathrm{M}}^{0} h^{2}\right)^{0.223} .
\end{aligned}
$$

Here we used the results from four data sets: $6 \mathrm{dF}$ Galaxy Redshift Survey measurements at efficient redshift $z_{\text {eff }}=0.1$ (Beutler et al. 2011), the SDSS DR7 BAO measurements at $z_{\mathrm{eff}}=0.35$ (Padmanabhan et al. 2012), the BOSS DR 9 measurements at $z_{\text {eff }}=0.57$ (Anderson et al. 2012), and WiggleZ measurements at higher redshift $z_{\text {eff }}=0.44,0.60,0.73$ (Blake et al. 2012).

The distance ratio vector is

$\boldsymbol{P}_{\mathrm{BAO}}^{\mathrm{obs}}=\left(\begin{array}{l}d_{0.1} \\ d_{0.35}^{-1} \\ d_{0.57}^{-1} \\ d_{0.44} \\ d_{0.60} \\ d_{0.73}\end{array}\right)=\left(\begin{array}{c}0.336 \\ 8.88 \\ 13.67 \\ 0.0916 \\ 0.0726 \\ 0.0592\end{array}\right)$.

The corresponding inverse covariance matrix is

$$
\boldsymbol{C}_{\mathrm{BAO}}^{-1}=\left(\begin{array}{cc}
I_{1} & 0 \\
0 & I_{2}
\end{array}\right)
$$

where

$$
\begin{aligned}
& \boldsymbol{I}_{1}=\left(\begin{array}{ccc}
4444.4 & 0 & 0 \\
0 & 34.602 & 0 \\
0 & 0 & 20.661157
\end{array}\right) \\
& \boldsymbol{I}_{2}=\left(\begin{array}{cccc}
24532.1 & -25 & 137.7 & 12099.1 \\
-25137.7 & 134598.4 & -64783.9 \\
12099.1 & -64783.9 & 128 & 837.6
\end{array}\right)
\end{aligned}
$$

The $\chi_{\mathrm{BAO}}^{2}$ value of the $\mathrm{BAO}$ can be expressed as

$$
\chi_{\mathrm{BAO}}^{2}=\Delta \boldsymbol{P}_{\mathrm{BAO}}^{\mathrm{T}} \boldsymbol{C}_{\mathrm{BAO}}^{-1} \Delta \boldsymbol{P}_{\mathrm{BAO}},
$$

where $\Delta \boldsymbol{P}_{\mathrm{BAO}}=\boldsymbol{P}_{\mathrm{BAO}}^{\mathrm{th}}-\boldsymbol{P}_{\mathrm{BAO}}^{\mathrm{obs}}$.

\subsection{CMB from WMAP 9 years}

We also used the WMAP 9 year data. We used the "WMAP distance priors" likelihood of three variables: the acoustic scale $l_{\mathrm{a}}$, the shift parameter $R$, and the recombination redshift $z_{*}$ to constrain parameters. They can be expressed as

$$
\begin{aligned}
& l_{\mathrm{a}}=\pi \frac{\int_{0}^{z_{*}} \frac{\mathrm{d} z}{E(z)} / H_{0}}{r_{s}\left(z_{*}\right)}, \\
& R=\frac{\Omega_{\mathrm{M} 0}^{1 / 2} H_{0}}{c} \int_{0}^{z_{*}} \frac{\mathrm{d} z}{E(z)},
\end{aligned}
$$

and the recombination redshift is given by $\mathrm{Hu} \&$ Sugiyama (1996),

$$
z_{*}=1048\left[1+0.00124\left(\Omega_{\mathrm{B}}^{0} h^{2}\right)^{-0.738}\left(1+g_{1}\left(\Omega_{\mathrm{M}}^{0} h^{2}\right)^{g_{2}}\right)\right],
$$

with

$$
\begin{aligned}
& g_{1}=0.0783\left(\Omega_{\mathrm{B}}^{0} h^{2}\right)^{-0.238}\left(1+39.5\left(\Omega_{\mathrm{B}}^{0} h^{2}\right)^{0.763}\right)^{-1}, \\
& g_{2}=0.560\left(1+21.1\left(\Omega_{\mathrm{B}}^{0} h^{2}\right)^{1.81}\right)^{-1} .
\end{aligned}
$$

The best-fit data are given by Hinshaw et al. (2013),

$\boldsymbol{P}_{\mathrm{CMB}}^{\mathrm{obs}}=\left(\begin{array}{c}l_{\mathrm{a}} \\ R \\ z_{*}\end{array}\right)=\left(\begin{array}{c}302.40 \\ 1.7246 \\ 1090.88\end{array}\right)$.

The corresponding inverse covariance matrix can be written as

$\boldsymbol{C}_{\mathrm{CMB}}{ }^{-1}=\left(\begin{array}{ccc}3.182 & 18.253 & -1.429 \\ 18.253 & 11887.879 & -193.808 \\ -1.429 & -193.808 & 4.556\end{array}\right)$

The $\chi_{\mathrm{CMB}}^{2}$ value of CMB is

$\chi_{\mathrm{CMB}}^{2}=\Delta \boldsymbol{P}_{\mathrm{CMB}}^{\mathrm{T}} \boldsymbol{C}_{\mathrm{CMB}}^{-1} \Delta \boldsymbol{P}_{\mathrm{CMB}}$,

where $\Delta \boldsymbol{P}_{\mathrm{CMB}}=\boldsymbol{P}_{\mathrm{CMB}}^{\mathrm{th}}-\boldsymbol{P}_{\mathrm{CMB}}^{\mathrm{obs}}$.

\section{Methods and results}

With the joint data, the total $\chi^{2}$ can be expressed as

$\chi^{2}\left(\delta, \Omega_{\mathrm{DM}}, w_{0}, w_{1}\right)=\chi_{\mathrm{SNe}}^{2}+\chi_{H}^{2}+\chi_{\mathrm{BAO}}^{2}+\chi_{\mathrm{CMB}}^{2}$.

The model parameters can be determined by computing the $\chi^{2}$ distribution. First, we calculated the lowest value of the total $\chi^{2} /$ d.o.f. $=0.990$ from simultaneous fitting. Then, we calculated the inverse covariance matrix to obtain the best-fit value $1 \sigma$ uncertainty $\delta=-0.022 \pm 0.006, \Omega_{\mathrm{DM}}^{0}=0.213 \pm 0.008, w_{0}=$ $-1.210 \pm 0.033$, and $w_{1}=0.872 \pm 0.072$.

To obtain the contour plot, we marginalized two of the four parameters to derive a new $\chi^{2}$ function depending on the other two parameters,

$\chi^{\prime 2}\left(\delta, \Omega_{\mathrm{DM}}\right)=\frac{1}{\Psi} \int_{w_{0}-\sigma_{w_{0}}}^{w_{0}+\sigma_{w_{0}}} \int_{w_{1}-\sigma_{w_{1}}}^{w_{1}+\sigma_{w_{1}}} \chi^{2}\left(\delta, \Omega_{\mathrm{DM}}, w_{0}, w_{1}\right) \mathrm{d} w_{0} \mathrm{~d} w_{1}$,

where $\Psi$ is the normalization factor to cause the $\chi^{\prime 2}$ to have the same lowest value as $\chi^{2}$. Then we used $\chi^{\prime 2}$ to derive the $\delta-\Omega_{\mathrm{DM}}$ $2 \mathrm{D}$ marginalized regions with different colors that represent $1 \sigma$ and $2 \sigma$ regions. Figure 1 shows the $\delta-\Omega_{\text {DM }}$ contours with different data combinations: SNe (gray and light-gray contours), $\mathrm{SNe}+\mathrm{BAO}$ (red and pink contours), $\mathrm{SNe}+\mathrm{CMB}$ (blue and light-purple contours), $\mathrm{CMB}+\mathrm{BAO}+H(z)$ (orange and yellow contours) and the full data-sets (black and cyan contours). This figure shows that the BAO data can set tight constraints on $\Omega_{\mathrm{DM}}$, while CMB can set tighter constraints on $\delta$ and $\Omega_{\mathrm{DM}}$. From Fig. 1, we find that the SNe data disagree with other data sets. This has been investigated by Nesseris \& Perivolaropoulos (2005) and Wei (2010a).

To test the reliability of our method, we also show the $w_{0}-w_{1}$ contours from $\mathrm{SNe}+\mathrm{BAO}+\mathrm{CMB}$ without the coupling $(\delta=0)$ with $1 \sigma$ region in black contours and $2 \sigma$ region in grey contours, which is presented in the left panel of Fig. 2. Our result is consistent with that of the WMAP team from comparing this figure with Fig. 10 of Hinshaw et al. (2013). The right panel of Fig. 2 shows the $w_{0}-w_{1}$ contours with coupling. We show the $\delta-w_{0}$ and $\Omega_{\mathrm{DM}}-w_{1}$ contours in Fig. 3 and the $\delta-w_{1}$ and $\Omega_{\mathrm{DM}}-w_{0}$ contours in Fig. 4. 


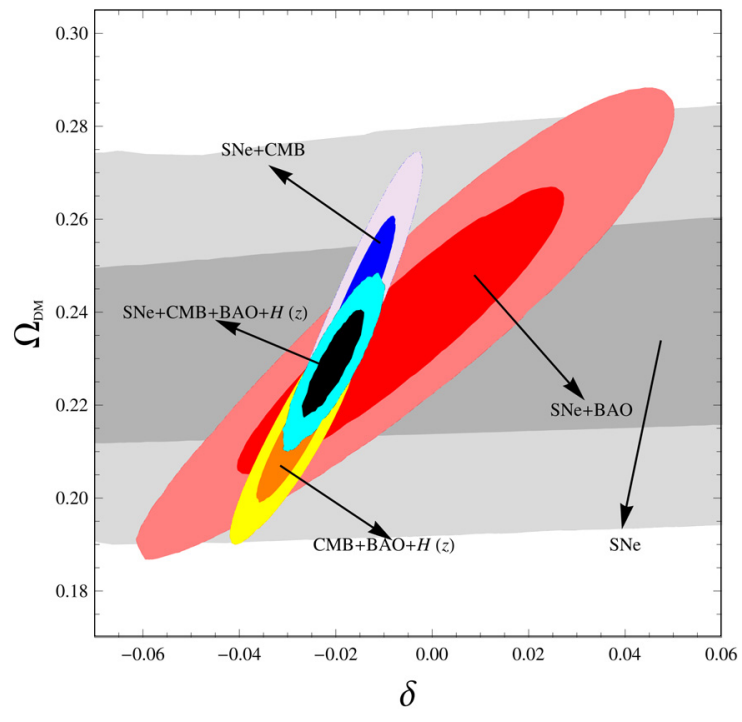

Fig. 1. $\delta-\Omega_{\mathrm{DM}}$ contours with different data combinations: $\mathrm{SNe}$ (gray and light-gray contours), $\mathrm{SNe}+\mathrm{BAO}$ (red and pink contours), $\mathrm{SNe}+$ $\mathrm{CMB}$ (blue and light-purple contours), $\mathrm{CMB}+\mathrm{BAO}+H(z)$ (orange and yellow contours), and $\mathrm{SNe}+\mathrm{CMB}+\mathrm{BAO}+H(z)$ (black and cyan contours). The central regions and the vicinity regions represent the $1 \sigma$ contours and $2 \sigma$ contours.
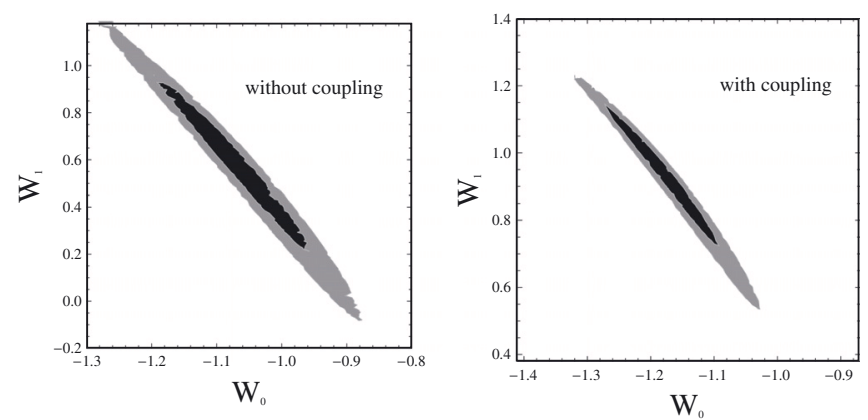

Fig. 2. Black and gray regions are $1 \sigma$ contours and $2 \sigma$ contours. The left panel shows $w_{0}$ vs. $w_{1}$ without coupling, the right panel shows $w_{0}$ vs. $w_{1}$ with coupling in our model.
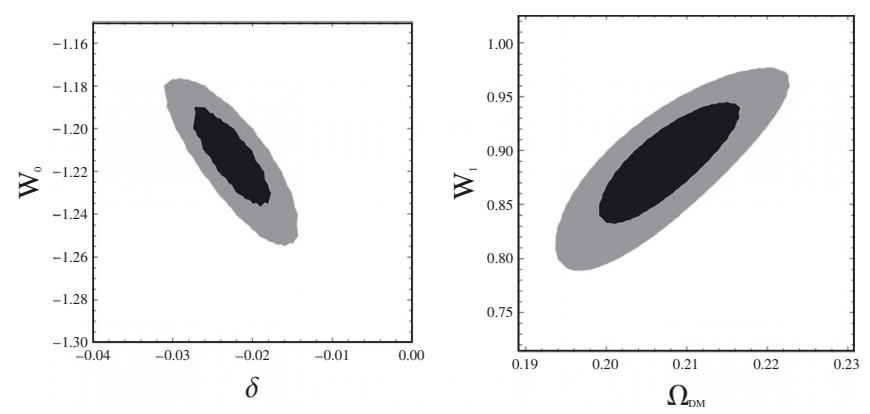

Fig. 3. Black and gray regions are $1 \sigma$ contours and $2 \sigma$ contours. The left panel shows $\delta$ vs. $w_{0}$, the right panel shows $\Omega_{\mathrm{DM}}$ vs. $w_{1}$.

From the best-fit parameters, the energy density evolution of DM and DE can be calculated. The ratio of DM density and DE density is

$\rho_{\mathrm{DM}} / \rho_{\mathrm{DE}}=\rho_{\mathrm{DM}}^{0} a^{-3+\delta} /\left(\rho_{\mathrm{DE}}^{\mathrm{NI}}(z)\left[1+\Theta\left(z, w_{0}, w_{1}, \delta\right)\right]\right)$.

Figure 5 shows the evolution of $\rho_{\mathrm{DM}} / \rho_{\mathrm{DE}}$ as a function of scale factor $a$ with best-fit parameters. The gray region represents
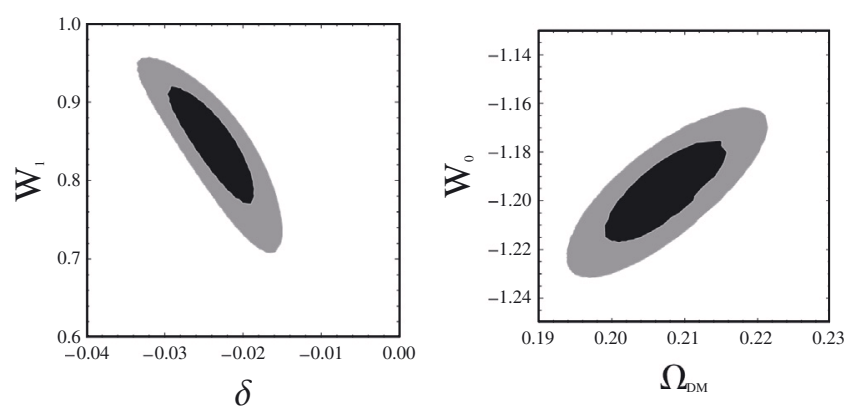

Fig. 4. Black and gray regions are $1 \sigma$ contours and $2 \sigma$ contours. The left panel shows $\delta$ vs. $w_{1}$, the right panel shows $\Omega_{\mathrm{DM}}$ vs. $w_{0}$.

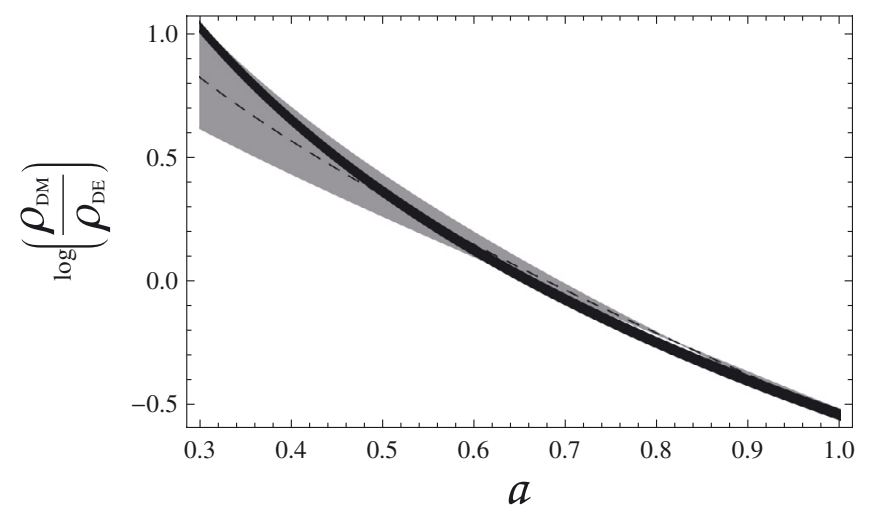

Fig. 5. Evolution of $\rho_{\mathrm{DM}} / \rho_{\mathrm{DE}}$ as a function of scale factor $a(z)$. The dashed line plots the interacting model with best-fit parameters, the gray region shows the $1 \sigma$ uncertainties. The black region represents the $\Lambda \mathrm{CDM}$ with uncertainties.

the $1 \sigma$ uncertainty in this model, the black one represents the $\Lambda \mathrm{CDM}$ case. In our model, $\delta<0$ means that the energy is transferred from dark matter to dark energy, which is consisted with Dalal et al. (2001) and Guo et al. (2007). Nevertheless, the energy density proportion evolves slower than in $\Lambda$ CDM case within $1 \sigma$ uncertainties when $a<0.5$, which means that our model can significantly help in reducing the coincidence problem.

The evolution of DE density plays an important role in solving the coincidence problem. Using Eq. (9) we can compute the DE evolution, which is shown in Fig. 6. The gray region above the black line shows that the DE density decreases within $1 \sigma$ when $a<0.5$, which can slow down the evolution of $\rho_{\mathrm{DM}} / \rho_{\mathrm{DE}}$, resulting in a good solution to the coincidence problem. However, the DE density evolves quite quickly in the very early stage of the Universe when $a<0.3$. The main reason is that DM mass transfer rate $\delta$ is assumed as a constant in our model.

Our Universe is currently undergoing an accelerating expansion. But in the very early time, the Universe was decelerating. This means that the evolution of deceleration parameter $q(z)$ is important, especially when $q\left(z_{\text {tr }}\right)=0, z_{\text {tr }}$ is the transition redshift. $q(z)$ can be expressed as

$q=-\frac{a \ddot{a}}{\dot{a}^{2}}=-1+\frac{1+z}{2 H(z)^{2}} \frac{\mathrm{d} H(z)^{2}}{\mathrm{~d} z}$

After substituting the best-fit parameters and their uncertainties in Eq. (38), we obtain $z_{\text {tr }}=0.63 \pm 0.07$. This value is slightly higher than those of Wang \& Dai (2006), Wang et al. (2007) and Abdel-Rahman \& Riad (2007) in $\Lambda$ CDM. The reason is the 


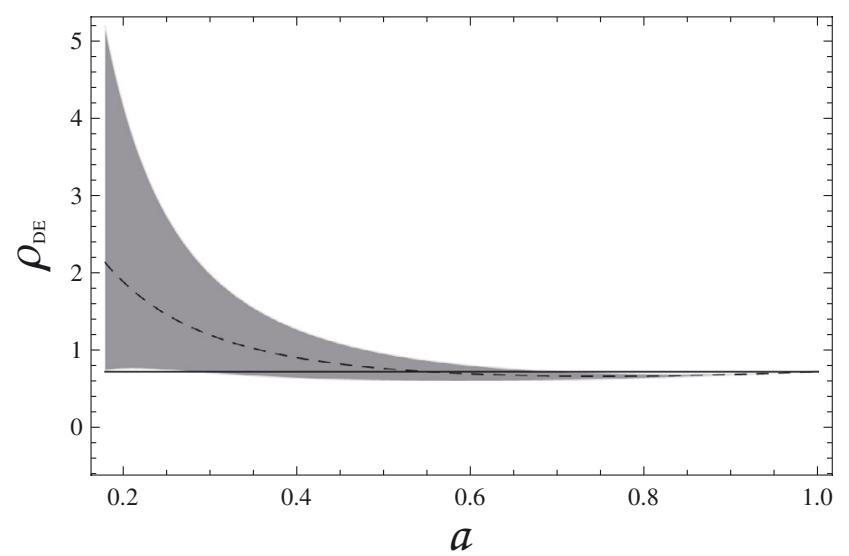

Fig. 6. Evolution of $\rho_{\mathrm{DE}}$ as a function of scale factor $a(z)$. The dashed line plots the interacting model with best-fit parameters, the gray region shows the $1 \sigma$ uncertainties. The black line represents the $\Lambda \mathrm{CDM}$ case.

energy transfer from DM to DE in our model. The DM density decreases quicker than that in the $\triangle \mathrm{CDM}$, while the DE density decreases much quicker in early times. Therefore a higher transition redshfit is needed for DE to resist gravitation.

\section{Conclusions and discussions}

We used the Union 2.1 SNe Ia, CMB from WMAP 9 years, BAO observation data from 6dFGRS, SDSS DR7, BOSS DR9, WiggleZ, and the latest Hubble parameter data to test the phenomenological interacting dark sector scenario with a dynamic $\operatorname{EoS} w_{\mathrm{DE}}(z)=w_{0}+w_{1} z /(1+z)$. We derived more stringent constraints on the phenomenological model parameters: $\delta=-0.022 \pm 0.006, \Omega_{\mathrm{DM}}^{0}=0.213 \pm 0.008, w_{0}=-1.210 \pm 0.033$ and $w_{1}=0.872 \pm 0.072$ with $\chi_{\min }^{2} /$ d.o.f. $=0.990$. From the contours using different data combinations in Fig. 1, we find that the $\mathrm{SNe}$ Ia data disagree with the combined CMB, BAO, and Hubble parameter data.

Our phenomenological scenario gives $\delta<0$ at $1 \sigma$ confidence level, which is consistent with Dalal et al. (2001) and Guo et al. (2007). This indicates that the energy is transferred from dark matter to dark energy. But the evolution of $\rho_{\mathrm{DM}} / \rho_{\mathrm{DE}}$ is slower than that in $\Lambda \mathrm{CDM}$ within $1 \sigma$ uncertainties, because $\rho_{\mathrm{DE}}$ decreases with scale factor $a$. Therefore our model represents a good approach to solve the coincidence problem.

The DE density evolves quickly in the very early epoch of the Universe, which is shown in Fig. 6. The main reason is that the value of $\delta$ is assumed to be constant in our model. In reality, the DM mass transfer rate $\delta(a)$ needs to be varied. We also derived the transition redshit $z_{\mathrm{tr}}=0.63 \pm 0.07$ in this model. Because of the interaction between DE and DM, the DE density decreases very quickly in early times, therefore a higher transition redshift is needed to resist gravitation.
Acknowledgements. We thank the anonymous referee for helpful comments and suggestions that have helped us improve our manuscript. This work is supported by the National Basic Research Program of China (973 Program, grant 2014CB845800) and the National Natural Science Foundation of China (grants 11373022, 11103007, 11033002 and J1210039).

\section{References}

Abdel-Rahman, A.-M. M., \& Riad, I. F. 2007, AJ, 134, 1391 Amendola, L. 2000, Phys. Rev. D, 62, 043511

Amendola, L., Campos, G. C., \& Rosenfeld, R. 2007, Phys. Rev. D, 75, 3506 Anderson, L., Aubourg, E., Bailey, S., et al. 2012, MNRAS, 427, 3435

Armendariz-Picon, C., Mukhanov, V., \& Steinhardt, P. J. 2001, Phys. Rev. D, 63, 3510

Bertolami, O., Gil Pedro, F., \& Le Delliou, M. 2007, Phys. Lett. B, 654, 165 Beutler, F., Blake, C., Colless, M., et al. 2011, MNRAS, 416, 3017 Blake, C., Brough, S., Colless, M., et al. 2012, MNRAS, 425, 405 Busca, N. G., Delubac, T., Rich, J., et al. 2013, A\&A, 552, A96

Cai, R.-G., \& Su, Q. 2010, Phys. Rev. D, 81, 103514

Caldwell, R. R. 2002, Phys. Lett. B, 545, 23

Cao, S., Liang, N., \& Zhu, Z.-H. 2011, Int. J. Mod. Phys. D, 22, 14

Chevallier, M., \& Polarski, D. 2001, Int. J. Mod. Phys. D, 10, 213

Chuang, C.-H., \& Wang, Y. 2013, MNRAS, 1955

Cole, S., Percival, W. J., Peacock, J., et al. 2005, MNRAS, 362, 505

Dalal, N., Abazajian, K., Jenkins, E., \& Manohar, A. V. 2001, Phys. Rev. L, 87, 141302

Del Campo, S., Herrera, R., Olivares, G., \& Pavón, D. 2006, Phys. Rev. D, 74, 023501

Eisenstein, D. J., \& Hu, W. 1998, ApJ, 496, 605

Eisenstein, D. J., Zehavi, I., Hogg, D. W., et al. 2005, ApJ, 633, 560

Farooq, O., \& Ratra, B. 2013, ApJ, 766, L7

Farrar, G. R., \& Peebles, P. J. E. 2004, ApJ, 604, 1

Feng, B., Wang, X., \& Zhang, X. 2005, Phys. Lett. B, 607, 35

Guo, Z.-K., Cai, R.-G., \& Zhang, Y.-Z. 2005, JCAP, 5, 2

Guo, Z.-K., Ohta, N., \& Tsujikawa, S. 2007, Phys. Rev. D, 76, 023508

Hinshaw, G., Larson, D., Komatsu, E., et al. 2013, ApJS, 208, 19

Hu, W., \& Sugiyama, N. 1996, ApJ, 471, 542

Linder, E. V. 2003, Phys. Rev. Lett., 90, 091301

Majerotto, E., Sapone, D., \& Amendola, L. 2004, unpublished [arXiv: astro-ph/0410543]

Moresco M., Cimatti, A., Jimenez, R., et al. 2012, JCAP, 8, 6

Nesseris, S., \& Perivolaropoulos, L. 2005, Phys. Rev. D, 72, 123519

Padmanabhan, N., Xu, X., Eisenstein, D. J., et al. 2012, MNRAS, 427, 2132

Percival, W. J., Reid, B. A., Eisenstein, D. J., et al. 2010, MNRAS, 401, 2148

Perlmutter, S., Aldering, G., Goldhaber, G., et al. 1999, ApJ, 517, 565

Planck Collaboration XVI. 2014, A\&A, in press, DOI: 10.1051/0004-6361/201321591

Ratra, B., \& Peebles, P. J. E. 1988, Phys. Rev. D, 37, 3406

Riess, A. G., Filippenko, A. V., Challis, P., et al. 1998, AJ, 116, 1009

Riess, A. G., Macri, L., Casertano, S., et al. 2011, ApJ, 730, 119

Rosenfeld, R. 2005, Phys. Lett. B, 624, 158

Sadjadi, H. M., \& Alimohammadi, M. 2006, Phys. Rev. D, 74, 103007

Simon, J., Verde, L., \& Jimenez, R. 2005, Phys. Rev. D, 71, 123001

Stern, D., Jimenez, R., Verde, L., Kamionkowski, M., \& Stanford, S. A. 2010, JCAP, 2, 8

Suzuki, N., Rubin, D., Lidman, C., et al. 2012, ApJ, 746, 85

Szydłowski M., 2006, Phys. Lett. B, 632, 1

Wang, F. Y. 2012, A\&A, 543, A91

Wang, F. Y., \& Dai, Z. G. 2006, MNRAS, 368, 371

Wang, F. Y., Dai, Z. G., \& Zhu, Z. H. 2007, ApJ, 667, 1

Wei, H. 2010a, Phys. Lett. B, 687, 286

Wei, H. 2010b, Phys. Lett. B, 691, 173

Wei, H., \& Cai, R.-G. 2006, Phys. Rev. D, 73, 083002

Zhang, C., Zhang, H., Yuan, S., Zhang, T.-J., \& Sun, Y.-C. 2012, ApJ, submitted [arXiv: 1207.4541] 